\title{
Analytical and Numerical Study of Soret and Dufour Effects on Double Diffusive Convection in a Shallow Horizontal Binary Fluid Layer Submitted to Uniform Fluxes of Heat and Mass
}

\author{
A. Lagra, ${ }^{1}$ M. Bourich, ${ }^{2}$ M. Hasnaoui $\left(\mathbb{D}^{1},{ }^{1}\right.$ A. Amahmid, ${ }^{1}$ and M. Er-Raki ${ }^{3}$ \\ ${ }^{1}$ Faculty of Sciences Semlalia, Department of Physics, LMFE, BP 2390, Marrakesh, Morocco \\ ${ }^{2}$ National School of Applied Sciences, Physics Department, LMFE, BP 575, Marrakesh, Morocco \\ ${ }^{3}$ High School of Technology, Cadi Ayyad University, LMFE, Essaouira, Morocco \\ Correspondence should be addressed to M. Hasnaoui; hasnaoui@uca.ac.ma
}

Received 30 August 2017; Accepted 17 December 2017; Published 12 February 2018

Academic Editor: Filippo de Monte

Copyright (C) 2018 A. Lagra et al. This is an open access article distributed under the Creative Commons Attribution License, which permits unrestricted use, distribution, and reproduction in any medium, provided the original work is properly cited.

\begin{abstract}
Combined Soret and Dufour effects on thermosolutal convection induced in a horizontal layer filled with a binary fluid and subject to constant heat and mass fluxes are investigated analytically and numerically. The thresholds marking the onset of supercritical and subcritical convection are predicted analytically and explicitly versus the governing parameters. The present investigation shows that different regions exist in the $N$-Du plane corresponding to different parallel flow regimes. The number, the extent, and the locations of these regions depend on whether $\mathrm{SrDu}>-\left(1+\mathrm{Le}^{2}\right) / 2 \mathrm{Le}^{2}=f(\mathrm{Le})$ or $\mathrm{SrDu}<-\left(1+\mathrm{Le}^{2}\right) / 2 \mathrm{Le}^{2}$. Conjugate effects of cross-phenomena on thresholds of fluid flow and heat and mass transfer characteristics are illustrated and discussed.
\end{abstract}

\section{Introduction}

Great interest in the study of thermosolutal convection in fluid and porous media has been motivated by its presence in many engineering applications, such as in hydrology, petrology, geophysics, and material processing technology where melting and solidification of binary alloys are involved [1]. More specifically, such flows are encountered in nature (lakes, solar ponds, and atmosphere). In the industrial field, examples include food processing, chemical processes, crystal growth, energy storage, material processing, and many other examples. Important experimental, analytical, and numerical results on convective heat and mass transfer are documented in earlier books of Nield and Bejan [2] and De Groot and Mazur [3]. Most studies on this topic are concerned with double diffusive convection in verti$\mathrm{cal} /$ horizontal cavities for which the flows induced by the buoyancy forces result from the imposition of both thermal and solutal boundary conditions on the vertical/horizontal walls [4-6].
The diffusion of mass due to temperature gradient is called Soret or thermodiffusion effect that often counts among the main drivers of various convective phenomena occurring within thermal stratified media. Recently, Rahman and Saghir [7] proposed a detailed historical review of works, focusing on different aspects of Soret effect. Examples of interesting phenomena resulting from the coupling between double diffusive convection and Soret effect are available in the paper by Bourich et al. [8] who investigated analytically and numerically Soret-driven thermosolutal convection within a shallow porous or fluid layer subject to a vertical gradient of temperature, using a Brinkman-Hazen-Darcy model in its transient form. The critical Rayleigh numbers for the onset of subcritical, oscillatory, and stationary convection were determined explicitly as functions of the governing parameters for infinite and finite layers.

Generally, Soret and Dufour effects are assumed negligible in problems related to double diffusive convection. However, such effects could be of significant effect when density differences exist in the flow regime. In fact, energy 
flux can be generated by composition of gradients (Dufour or diffusion-thermoeffect). Similarly, mass fluxes can be created by temperature gradients (Soret or thermodiffusion effect). In an earlier study, Malashetty [9] investigated the effect of anisotropic thermoconvective currents, in the presence of Soret and Dufour effects, on the critical Rayleigh number for both marginal and overstable motions. Mortimer and Eyring [10] used the elementary transition state approach to obtain a simple model theory for the Soret and Dufour effects. They found that the results of the theory conform to the Osanger reciprocal relationship. Gaikwad et al. [11] studied the onset of double diffusive convection in a twocomponent couple stress fluid layer with Soret and Dufour effects using both linear and nonlinear stability analysis. The effects of Soret and Dufour parameters together with the couple stress parameter on the stationary and oscillatory convection are graphically illustrated and discussed. In the presence of Soret and Dufour effects, Nithyadevi and Yang [12] presented numerical results on natural convection in a square enclosure filled with water, partially heated from one vertical wall, and totally cooled from the opposite vertical wall. The study was conducted around the maximum density for three different combinations of the heating element location. Makinde et al. [13] described a theoretical study used to analyze the hydromagnetic flow and mass diffusion of chemical reactive species with first- and higher-order reactions of an electrically conducting fluid over a moving vertical plate. The study was conducted in the presence of Soret and Dufour effects with convective heat exchange at the plate surface. Pal and Mondal [14] considered the problem of steady laminar, hydromagnetic two-dimensional mixed convection flow due to stretching sheet in the presence of Soret and Dufour effects. Cheng [15] examined the Dufour and Soret effects on the steady boundary layer flow due to natural convection heat and mass transfer over a vertical cone embedded in a porous medium with constant wall temperature and concentration. The results presented show that the effects of Dufour and Soret parameters on the local surface temperature are increased by increasing the Lewis number. Tsai and Huang [16] investigated heat and mass transfer from natural convection flow along a vertical surface with variable heat fluxes embedded in a porous medium due to Soret and Dufour effects. They concluded that Soret and Dufour effects could play a significant role. Soret and Dufour effects have been also considered by Hayat et al. [17] who studied mixed convection boundary layer flow about a linearly stretching vertical surface in a porous medium filled with a viscoelastic fluid and, more recently, by Wang et al. [18] who studied the onset of double diffusive convection in a horizontal cavity.

The main purpose of the present investigation is to study analytically and numerically the combined effects of Soret and Dufour parameters on double diffusive convection developed in a horizontal layer filled with a binary fluid. This paper is an extended version of preliminary results presented in a conference [19]. Analytical predictions are developed and validated numerically for shallow enclosures. The Dufour parameter effects on thresholds of stationary convection, subcritical convection, flow structure, and heat and mass transfer are also discussed.

\section{Mathematical Formulation}

The system under study is a two-dimensional shallow cavity of length $L^{\prime}$ and height $H^{\prime}$, filled with a binary fluid. The vertical end-walls of the layer are adiabatic and impermeable to mass transfer while its horizontal walls are subject to uniform fluxes of heat, $q^{\prime}$, and mass, $j^{\prime}$. The flow is assumed to obey the Boussinesq approximation. Using the vorticity and the stream function formulation and taking into account the cross-phenomena (Soret and Dufour effects), the dimensionless governing equations are obtained as follows:

$$
\begin{aligned}
& \frac{1}{\operatorname{Pr}}\left[\frac{\partial\left(\nabla^{2} \psi\right)}{\partial t}+u \frac{\partial\left(\nabla^{2} \psi\right)}{\partial x}+v \frac{\partial\left(\nabla^{2} \psi\right)}{\partial y}\right] \\
& =\nabla^{4} \psi-R_{T}\left(\frac{\partial T}{\partial x}+N \frac{\partial S}{\partial x}\right), \\
& \frac{\partial T}{\partial t}+u \frac{\partial T}{\partial x}+v \frac{\partial T}{\partial y}=\nabla^{2} T+D_{u} N \nabla^{2} S, \\
& \frac{\partial S}{\partial t}+u \frac{\partial S}{\partial x}+v \frac{\partial S}{\partial y}=\frac{S r}{N} \nabla^{2} T+\frac{1}{L e} \nabla^{2} S, \\
& \nabla^{2} \psi=-\zeta .
\end{aligned}
$$

The associated hydrodynamic, thermal, and solutal boundary conditions are

$$
\begin{aligned}
x= \pm \frac{A_{r}}{2}: \quad & \psi=0, \\
\frac{\partial T}{\partial x} & =0, \\
\frac{\partial S}{\partial x} & =0, \\
y= \pm \frac{1}{2}: \quad & \psi=0, \\
& \frac{\partial T}{\partial y}=-\frac{1-\mathrm{NDu}}{1-\mathrm{LeSrDu}}=-\varphi_{T}, \\
\frac{\partial S}{\partial y} & =-\frac{1-\mathrm{LeSr} / N}{1-\mathrm{LeSrDu}}=-\varphi_{S} .
\end{aligned}
$$

The parameters governing the problem are the thermal Darcy-Rayleigh number, $R_{T}=g \beta_{T} \Delta T^{\prime} H^{\prime 3} /\left(\kappa_{11} \nu\right)$, the Prandlt number, $\operatorname{Pr}=\nu / \kappa_{11}$, the Lewis number, Le $=\kappa_{11} / \kappa_{22}$, the buoyancy ratio, $N=\beta_{S} \Delta S^{\prime} /\left(\beta_{T} \Delta T^{\prime}\right)$, and the aspect ratio of the cavity, $A_{r}=L^{\prime} / H^{\prime}$. The parameters $\mathrm{Sr}$ and $\mathrm{Du}$ are, respectively, the Soret and Dufour parameters expressed as $\mathrm{Sr}=\beta_{S} \mathrm{~K}_{21} /\left(\beta_{T} \mathrm{~K}_{11}\right)$ and $\mathrm{Du}=\beta_{T} \mathrm{~K}_{12} /\left(\beta_{S} \mathrm{~K}_{11}\right)$, with $\kappa_{11}$ being the thermal diffusivity, $\kappa_{12}$ being cross-diffusion 
due to solute concentration component, $\kappa_{21}$ being crossdiffusion due to temperature component, and $\kappa_{22}$ being solute diffusivity.

In the presence of the cross-diffusion phenomena, the Nusselt and Sherwood numbers are defined as follows:

$$
\begin{aligned}
\mathrm{Nu} & =\frac{1}{\Delta T+N \mathrm{Nu} \Delta S}, \\
\mathrm{Sh} & =\frac{1}{\Delta S+((\mathrm{Sr} \cdot \mathrm{Le}) / N) \Delta T},
\end{aligned}
$$

where $\Delta T=T(0,-1 / 2)-T(0,1 / 2)$ and $\Delta S=S(0,-1 / 2)-$ $S(0,1 / 2)$ are the temperature and concentration differences, evaluated at $x=0$ with the origin of the coordinate system being taken at the center of the cavity.

\section{Methods}

3.1. Numerical Solution. The numerical solution of (1) to (3) was obtained using a finite-difference method, described in detail by Bourich et al. [8]. A second-order scheme was used for the discretization of the spatial derivatives. Equations (2)-(3) were marched in time using the Alternating Direction Implicit (ADI) method. The stream function equation (4) was solved at each time step with the Point Successive Overrelaxation (PSOR) method with an optimum overrelaxation coefficient calculated for the used grid. In addition, a convergence criterion was adopted for the stream function to satisfy a variation by less than $10^{-5}$ for each time step. A second criterion $\sum \sum \mid \Gamma_{i, j}^{n+1}-$ $\Gamma_{i, j}^{n}\left|/ \sum \sum\right| \Gamma_{i, j}^{n+1} \mid \leq 10^{-6}$ was used to check the convergence of the numerical code. Here, $\Gamma$ stands for any of the variables $T, S$, or $\psi$. The superscripts $n$ and $(n+1)$ indicate the iterations numbers, and the subscripts $i$ and $j$ indicate locations in the grid system. For large aspect ratio enclosures, nonuniform grid was used in the $x$ direction near the short walls to capture the flow details near the enclosures end-walls and also in the $y$-direction to obtain a finer grid in the close vicinity of the horizontal walls.

3.2. Analytical Solution. For a shallow enclosure with constant heat and mass flux boundary conditions, an approximate analytical solution based on the parallel flow concept is possible, which renders the problem amenable to a parametric study while retaining the essential physics of the problem. The analytical solution is developed for steady-state flows using the parallel flow approximation (see, e.g., Bourich et al. [8]), which leads to the following simplifications (justified by examining the streamlines, isotherms, and iso-concentration lines obtained numerically). These simplifications lead to $\psi(x, y)=\psi(y), T(x, y)=C_{T} x+\theta_{T}(y)$, and $S(x, y)=$ $C_{S} x+\theta_{S}(y)$, where $C_{T}$ and $C_{S}$ are, respectively, unknown constant temperature and concentration gradients in the $x$ direction (the direction of the long sides of the cavity). Using these approximations together with the boundary conditions (5a) and (5b), (1)-(3) reduceto a set of ordinary differential equations for which the solution is obtained as follows:

$$
\begin{aligned}
& \psi(y)=\psi_{0}\left(4 y^{2}-1\right)^{2} \\
& T(x, y) \\
& =C_{T} x+\frac{C_{T}-C_{S} \operatorname{DuNLe}}{1-\operatorname{LeSrDu}} \frac{\psi_{0}}{15} y\left[48 y^{4}-40 y^{2}+15\right] \\
& \quad-\varphi_{T} y \\
& S(x, y) \\
& =C_{S} x+\frac{C_{S}-C_{T} \operatorname{Sr} / N}{1-\operatorname{LeSrDu}} \cdot \operatorname{Le} \frac{\psi_{0}}{15} \\
& \quad \cdot y\left[8 y^{4}-40 y^{2}+15\right]-\varphi_{S} y
\end{aligned}
$$

where $\psi_{0}$ is the stream function value at the midheight of the layer, given by

$$
\psi_{0}=\frac{R_{T}}{384}\left(C_{T}+N C_{S}\right)
$$

The analytical expressions of $C_{T}$ and $C_{S}$ were determined by using thermal and solutal balances in the layer, which leads to the following expressions:

$$
\begin{aligned}
C_{T} & =-\frac{8}{15} \psi_{0} \\
& \cdot \frac{2 a \psi_{0}^{2}\left(\varphi_{T}+\varphi_{S} N D u\right) \operatorname{Le}^{2}+A\left(\varphi_{T}-\varphi_{S} \operatorname{LeNDu}\right)}{\left(2 a \psi_{0}^{2}\right)^{2}+2 a \psi_{0}^{2} B+A^{2}}, \\
C_{S} & =-\frac{8}{15} \psi_{0} \\
& \cdot \frac{2 a \psi_{0}^{2}\left(\varphi_{T}(\operatorname{LeSr} / N)+\varphi_{S}\right) \operatorname{Le}+A \operatorname{Le}\left(\varphi_{S}-\varphi_{T}(\mathrm{Sr} / N)\right)}{\left(2 a \psi_{0}^{2}\right)^{2}+2 a \psi_{0}^{2} B+A^{2}},
\end{aligned}
$$

where $a=64 / 315, A=1-\mathrm{LeSrDu}$, and $B=\mathrm{Le}^{2}+2 \mathrm{Le}^{2} \mathrm{DuSr}+$ 1.

Introducing the expressions of $C_{T}$ and $C_{S}$ into (8) yields a fourth-order polynomial in terms of $\psi_{0}$ for which the following solutions are obtained:

$$
\begin{aligned}
& \psi_{0}=0, \\
& \psi_{0}=\mp \frac{1}{2}\left[-d_{1} \mp \sqrt{d_{1}^{2}-d_{2}}\right]^{1 / 2},
\end{aligned}
$$

with

$$
\begin{aligned}
d_{1} & =\frac{1}{2 a \mathrm{Le}^{2}}\left[B-\frac{R_{T}}{720} F_{1}\right], \\
d_{2} & =\frac{1}{a^{2} \mathrm{Le}^{2}}\left[A^{2}-\frac{R_{T}}{720} F_{2}\right], \\
F_{1} & =\operatorname{Le}(N+\mathrm{Le}), \\
F_{2} & =N C_{1}+C_{2},
\end{aligned}
$$

where $C_{1}=\operatorname{Du}[\operatorname{Le}(\operatorname{Sr}-1)-1]+\operatorname{Le}$ and $C_{2}=\operatorname{SrLe}[\operatorname{Le}(\mathrm{Du}-$ 1) -1$]+1$. 
From a mathematical point of view, (10) may exhibit, in addition to the rest state solution, two types of bifurcations depending on the sign within the square root. Although it should be mentioned that several numerical tests were performed, only the solutions corresponding to positive sign were obtained numerically. Hence, the convective solution with the negative sign within the square root is termed "unstable solution" and the convective solution corresponding to positive sign within the square root is similarly qualified "stable solution."

The new expressions of Nusselt and Sherwood numbers are obtained as follows:

$$
\begin{aligned}
\mathrm{Nu} & =\frac{1}{1-(8 / 15) \psi_{0} C_{T}}, \\
\mathrm{Sh} & =\frac{1}{1-(8 / 15) \psi_{0} \mathrm{LeC}_{S}} .
\end{aligned}
$$

Foremost, it may be remarked from (10) that the parallel flow solutions exist only when the following two conditions are satisfied:

$$
\begin{array}{r}
-d_{1} \mp \sqrt{d_{1}^{2}-d_{2}}>0, \\
d_{1}^{2}-d_{2}>0 .
\end{array}
$$

The resolution of the inequalities of (13) is performed in the $N$-Du plane with $\mathrm{Sr}$, Le, and $R_{T}$ as parameters. Depending of the sign of $B$ (parameter in the expression of $d_{1}$ given before), two main cases are possible.

3.2.1. The Parameter $B>O$. This condition is satisfied if $\mathrm{SrDu}>-\left(1+\mathrm{Le}^{2}\right) / 2 \mathrm{Le}^{2}$. Depending on the signs of $F_{1}$ and $F_{2}$, four cases are to be distinguished.

Case $1\left(F_{1} \leq 0\right.$ and $\left.F_{2} \leq 0\right)$. For this case, $F_{1} \leq 0 \Rightarrow d_{1} \geq 0$ and $F_{2} \leq 0 \Rightarrow d_{2} \geq 0$, which means that the parallel flow solution is not existing regardless the values of Sr, Le and $R_{T}$. In Figure 1 , the domain in the $N$-Du plane where the convective parallel flow is not possible is denoted as region 1. This region is defined by $F_{1} \leq 0$ and $F_{2} \leq 0$.

Case $2\left(F_{1} \leq 0\right.$ and $\left.F_{2}>0\right)$. For this case, $F_{1} \leq 0 \Rightarrow d_{1} \geq 0$ and $F_{2}>0 \Rightarrow d_{2}<0$ for $R_{T} \geq R_{T C}=R_{0}\left(A^{2} / F_{2}\right)$. Thus, the unstable solution is not possible and the stable solution is possible only when $R_{T}$ exceeds the critical value $R_{T C}$. The convective flow bifurcates from the rest state through a zero amplitude convection which indicates that the solution corresponds to a supercritical bifurcation. The critical value of $R_{T C}$ represents the supercritical Rayleigh number, $R_{T C}^{\text {sup }}$, marking the onset of the supercritical convection. The expression of this threshold value is given by

$$
R_{T C}^{\text {sup }}=R_{0} \frac{A^{2}}{F_{2}} \text {. }
$$

In Figure 1, the domain in the $N$-Du plane for which the conditions of Case 2 are satisfied is denoted as region 2 and it is defined by $F_{1} \leq 0$ and $F_{2}>0$.
Case $3\left(F_{1}>0\right.$ and $\left.F_{2}>0\right)$. For these conditions, the signs of $d_{1}$ and $d_{2}$ depend on the Rayleigh number as follows:

$$
\begin{aligned}
& d_{1} \geq 0 \quad \text { for } R_{T} \leq R_{T C_{1}}=R_{0} \frac{B}{F_{1}} \\
& d_{2} \geq 0 \quad \text { for } R_{T} \leq R_{T C_{2}}=R_{0} \frac{A^{2}}{F_{2}} .
\end{aligned}
$$

As a result, two subcases are to be considered.

Case $3.1\left(R_{T_{2}} \leq R_{T C_{1}}\right)$. The development of the above inequality leads to

$$
F_{3}=N\left(A^{2} \mathrm{Le}-B C_{1}\right)+A^{2} \mathrm{Le}^{2}-B C_{2} \leq 0 .
$$

Note that, for $R_{T} \leq R_{T C_{2}}, d_{1} \geq 0$ and $d_{2} \geq 0$ which means that there is no parallel flow solution. On the other hand, for $R_{T}>R_{T_{2}}, d_{1} \geq 0$ and $d_{2}<0$ indicating the existence of a supercritical bifurcation and the corresponding supercritical Rayleigh number is given by (14). The domain corresponding to these conditions in the $N$-Du plane is denoted region 3 in Figure 1. This region is defined by $F_{1}>0, F_{2}>0$, and $F_{3}=N\left(A^{2} \mathrm{Le}-B C_{1}\right)+A^{2} \mathrm{Le}^{2}-B C_{2} \leq 0$.

Even if regions 2 and 3 seem to have similar characteristics, they are presented separately because they differ in terms of some asymptotical behaviors at large values of $R_{T}$.

Case $3.2\left(R_{T C_{2}}>R_{T C_{1}}\right)$. For this case $F_{3}=N\left(A^{2}\right.$ Le $\left.-B C_{1}\right)+$ $\left.A^{2} \mathrm{Le}^{2}-B C_{2}>0\right)$. Hence, for $R_{T}<R_{T C_{1}}, d_{1} \geq 0$ and $d_{2} \geq 0$. It follows that there is no parallel flow solution. For $R_{T C_{1}}<$ $R_{T} \leq R_{T C_{2}}, d_{1}<0$ and $d_{2} \geq 0$ which means that both stable and unstable solutions are existing within this range of $R_{T}$. The stable solution corresponds to a subcritical bifurcation, which occurs through finite amplitude convection at a saddle node point. The subcritical threshold, $R_{T C}^{\text {sub }}$, is given by

$$
\begin{aligned}
& R_{T C}^{\text {sub }} \\
& =R_{0} \frac{\left(B F_{1}-2 \mathrm{Le}^{2} F_{2}\right)+2 \mathrm{Le} \sqrt{A^{2} F_{1}^{2}+\mathrm{Le}^{2} F_{2}^{2}-B F_{1} F_{2}}}{F_{1}^{2}} .
\end{aligned}
$$

In addition, for $R_{T}>R_{T C_{2}}, d_{1}<0$ and $d_{2}<0$ which implies that the unstable solution disappears for this range of $R_{T}$. The domain corresponding to these conditions in the $N$ Du plane is termed region 4 in Figure 1 . This region is defined by $F_{1}>0, F_{2}>0$, and $F_{3}=N\left(A^{2} \mathrm{Le}-B C_{1}\right)+A^{2} \mathrm{Le}^{2}-B C_{2}>0$.

Case $4\left(F_{1}>0\right.$ and $\left.F_{2}<0\right)$. For these conditions, $d_{1} \geq 0$ for $R_{T} \leq R_{T C_{1}}=R_{0}\left(B / F_{1}\right)$ and $d_{2} \geq 0$ independently of the values of the Rayleigh number. It follows that two subcases are possible.

Case $4.1\left(R_{T} \leq R_{T C_{1}}\right)$. Note that, for this range of $R_{T}, d_{1} \geq 0$ and $d_{2} \geq 0$ which means that there is no parallel flow solution.

Case $4.2\left(R_{T}>R_{T C_{1}}\right)$. This condition is satisfied for $d_{1}<$ 0 and $d_{2} \geq 0$. This means that both stable and unstable 


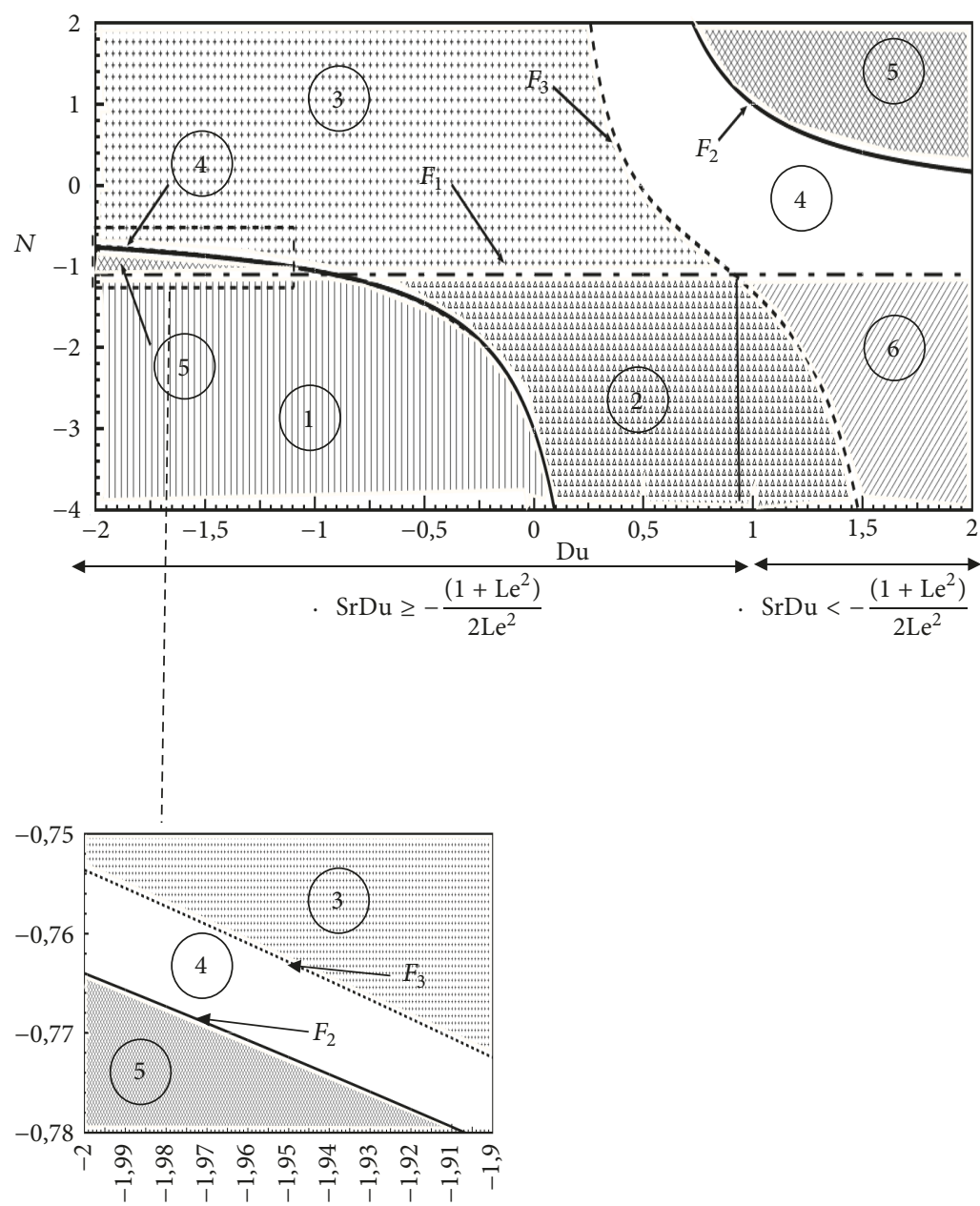

FIGURE 1: Regions corresponding to different bbehaviours for $\mathrm{Sr}=0.1$ and $\mathrm{Le}=1.1$.

solutions are existing for any value of $R_{T}$ greater than the subcritical threshold, $R_{T C}^{\text {sub }}$, given by the same expression as that for region 4 . The corresponding domain in the $N$-Du plane is termed region 5 in Figure 1 and it is defined by $F_{1}>0$ and $F_{2}<0$.

3.2.2. The Parameter $B<O$. This inequality is satisfied for $\mathrm{SrDu}<-\left(1+\mathrm{Le}^{2}\right) / 2 \mathrm{Le}^{2}$. For this case also, four cases are possible depending of the signs of $F_{1}$ and $F_{2}$.

Case $1\left(F_{1}>0\right.$ and $\left.F_{2}<0\right)$. These conditions are the same as those defining region 5 . The corresponding domain in the $N$-Du plane is termed region 5 in Figure 1 and it is defined by $F_{1}>0$ and $F_{2}<0$.

Case $2\left(F_{1}>0\right.$ and $\left.F_{2} \geq 0\right)$. For these conditions, $d_{1}<0$ independently of the values of the Rayleigh number and $d_{2} \geq$ 0 for $R_{T} \leq R_{T C_{2}}=R_{0}\left(A^{2} / F_{2}\right)$. This means that both stable and unstable solutions are existing in this range for $R_{T} \geq R_{T C}^{\text {sub }}$. The supercritical threshold Rayleigh number is given by (17). In addition, for $R_{T}>R_{T C_{2}}, d_{1}<0$, and $d_{2}<0$ which implies that the unstable solution disappears for this range of $R_{T}$. The corresponding domain in the $N$-Du plane is termed region 4 in Figure 1, defined by $F_{1}>0$ and $F_{2} \geq 0$.

Case $3\left(F_{1} \leq 0\right.$ and $\left.F_{2} \geq 0\right)$. For these conditions, the signs of $d_{1}$ and $d_{2}$ depend on the Rayleigh number as follows:

$$
\begin{aligned}
& d_{1} \geq 0 \quad \text { for } R_{T} \geq R_{T C_{1}}=R_{0} \frac{B}{F_{1}}, \\
& d_{2} \geq 0 \text { for } R_{T} \leq R_{T C_{2}}=R_{0} \frac{A^{2}}{F_{2}} .
\end{aligned}
$$

Two subcases emerge from these conditions.

Case $3.1\left(R_{T C_{2}} \leq R_{T C_{1}}\right)$. This inequality is verified for $F_{3}=$ $N\left(A^{2} \mathrm{Le}-B C_{1}\right)+A^{2} \mathrm{Le}^{2}-B C_{2} \geq 0$. Thus, for $R_{T} \leq R_{T C_{2}}, d_{1} \leq 0$ and $d_{2} \geq 0$, which means that both stable and unstable solutions are existing. The bifurcation is of a subcritical nature and the subcritical Rayleigh number is given by (17). For $R_{T}>$ $R_{T_{2}}$, the unstable solution disappears within this range of $R_{T}$. The domain in the $N$-Du plane satisfying these conditions is indicated by region 6 in Figure 1. This region is defined by $F_{1} \leq 0, F_{2} \geq 0$, and $F_{3} \geq 0$. It is to underline that, even if 
regions 5 and 6 seem to have the same characteristics, they are presented separately because they differ in terms of some asymptotical behaviors at large values of $R_{T}$.

Case $3.2\left(R_{T C_{2}}>R_{T C_{1}}\right)$. For this case, $F_{3}<0$. Then, for $R_{T}<$ $R_{T C_{1}}, d_{1} \geq 0$ and $d_{2} \geq 0$ and the parallel flow solution is not existing. On the other side, for $R_{T}>R_{T C_{2}}, d_{1} \geq 0$ and $d_{2}<0$ indicating the existence of a supercritical bifurcation and the corresponding supercritical Rayleigh number is given by (14). The domain corresponding to these conditions in the $N$-Du plane is termed region 2 in Figure 1 and it is defined by $F_{1} \leq 0, F_{2} \geq 0$, and $F_{3}<0$.

Case $4\left(F_{1} \leq 0\right.$ and $\left.F_{2} \leq 0\right)$. For this case, the inequality $\mathrm{SrDu}<-\left(1+\mathrm{Le}^{2}\right) / 2 \mathrm{Le}^{2}$ is verified and there is no corresponding domain in the $\mathrm{N}$-Du plane.

In summary, the $N$-Du plane can be divided into five regions for $\mathrm{SrDu}>-\left(1+\mathrm{Le}^{2}\right) / 2 \mathrm{Le}^{2}$ and into four regions for $\mathrm{SrDu}<-\left(1+\mathrm{Le}^{2}\right) / 2 \mathrm{Le}^{2}$. In region 1 , the parallel flow is not possible while in regions 2 and 3, the only possible flow is the supercritical stationary one. For the remaining regions (regions 4, 5, and 6) both subcritical and supercritical convections are possible.

\section{Results and Discussion}

4.1. Effect of Du and $N$ on Convection Thresholds. This section is devoted to analyze the combined effects of the Dufour parameter and the buoyancy ratio on thresholds of convection. In addition, some interesting behaviors of the fluid flow are illustrated and discussed.

The parameter Du represents the relative importance of the cross-diffusion due to solute concentration component with respect to that due to the thermal gradient. It could be varied by changing the mass flux intensity or by considering various working binary fluids. For a given $N$, different regions could be crossed in Figure 1 by incrementing Du, depending on whether $N \geq N_{L}^{\text {Sup }}$ or $N<N_{L}^{\text {Sup }}$. The parameter $N_{L}^{\text {Sup }}$ is calculated analytically and its expression is given by $N_{L}^{\text {Sup }}=$ $-\mathrm{SrLe}^{2} /(\mathrm{Le}(\mathrm{Sr}-1)-1)$. The effect of Du on thresholds of stationary convection is exemplified in Figures 2(a) and 2(b) illustrating, respectively, the cases $N \geq N_{L}^{\text {Sup }}$ (case illustrated with $N=1.5$, Le $=1.1$, and different Sr) and $N<N_{L}^{\text {Sup }}$ (case illustrated with $N=-1.5$, Le $=1.1$, and different $\mathrm{Sr}$ ). The value $\mathrm{Le}=1.1$ was chosen within the experimental range of the Lewis number where both cross-phenomena of Soret and Dufour effects are significant.

For $N \geq N_{L}^{\text {Sup }}$, the threshold of stationary convection is characterized by a decrease toward a minimum (well visible in Figure 2(a) for the negative values of Sr but not visible for the positive values of this parameter due to the restricted range of Du). It is seen in Figure 2(a) that the threshold of stationary convection decreases first by increasing $\mathrm{Du}$ to reach a minimum value at $\mathrm{Du}=\mathrm{Du}_{\min }$ and increases afterwards quickly toward a vertical asymptote (infinite value) for $\mathrm{Du}=$ $\mathrm{Du}_{C}^{\text {sup }}$. However, for the positive values of $\mathrm{Sr}$, the variations of

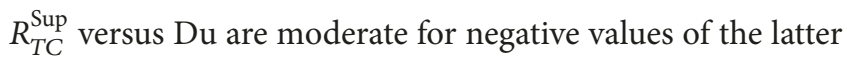
parameter. The increase of $R_{T C}^{\text {Sup }}$ accompanying the increase of $\mathrm{Du}$ in its negative range is characterized by an augmentation which becomes more and more slow by decreasing $\mathrm{Sr}$ within its positive range. In the positive range of $\mathrm{Du}$, the fast increase toward the vertical asymptote is also observed for the positive values of Sr. The critical value of Du leading to this asymptote increases by decreasing Sr. For $N<N_{L}^{\text {Sup }}$, an opposite behavior to that described in Figure 2(a) is observed. In fact, an increase of $\mathrm{Du}$ from the limiting value $\mathrm{Du}_{C}$ sup induces first a sharp decrease of $R_{T C}^{\text {sup }}$ from infinite toward a minimum value obtained at $\mathrm{Du}=\mathrm{Du}_{\min }$. Afterward, $R_{T C}^{\text {sup }}$ increases slowly (with different rates depending on $\mathrm{Sr}$ ). These results show that, for $N \geq N_{L}^{\text {Sup }} /\left(N<N_{L}^{\text {Sup }}\right)$, the Dufour parameter could play a destabilizing effect if $\mathrm{Du}<\mathrm{Du}_{\mathrm{C}}^{\text {sup }} /\left(\mathrm{Du}_{\mathrm{C}}^{\text {sup }}<\mathrm{Du}<\right.$ $\left.\mathrm{Du}_{\text {min }}\right)$ or a stabilizing one if $\mathrm{Du}_{\text {min }}<\mathrm{Du}<\mathrm{Du}_{\mathrm{C}}^{\text {sup }} /(\mathrm{Du}<$ $\left.\mathrm{Du}_{\text {min }}\right)$. The analytical expressions of $\mathrm{Du}_{\min }$ and $\mathrm{Du}_{C}^{\text {sup }}$ are, respectively, given by

$$
\begin{aligned}
& \operatorname{Du}_{C}^{\text {sup }}=\frac{\operatorname{SrLe}(\mathrm{Le}+1)-N \mathrm{Le}-1}{\operatorname{SrLe}(N+\mathrm{Le})-N(\mathrm{Le}+1)} \\
& \operatorname{Du}_{\text {min }} \\
& =-\frac{\operatorname{LeSr}(-2 \operatorname{SrLe}(\mathrm{Le}+1)+N(2 \mathrm{Le}+1)+\mathrm{Le}+2)-N(\mathrm{Le}+1)}{\operatorname{LeSr}(\operatorname{LeSr}(N+\mathrm{Le})-N(\mathrm{Le}+1))} .
\end{aligned}
$$

The Dufour effect on $R_{T C}^{\text {Sub }}$ is illustrated for different values of Soret parameter, Sr, for the three possible cases (determined analytically) that lead to different behaviors depending on the crossing region. Thus, Figure 3(a) illustrates the case $N_{L}^{\text {Sub }} \geq N>$-Le, Figure 3(b) illustrates the case $N>$ $N_{L}^{\text {Sub }}$, and the third case illustrated in Figure 3(c) corresponds to $N \leq-$ Le and $\mathrm{Sr}<\mathrm{Sr}_{C}$. The analytical expression of $N_{L}^{\text {Sub }}$ is given by

$$
N_{L}^{\mathrm{Sub}}=\frac{\mathrm{Sr} \cdot \mathrm{Le}^{2}}{\mathrm{Le} \cdot(2-\mathrm{Sr})+2} .
$$

For the first case, Figure 3(a) shows that the subcritical Rayleigh number, $R_{T C}^{\text {Sub }}$, decreases linearly by increasing Du and vanishes when $\mathrm{Du}$ approaches a critical value $D_{\mathrm{C}}^{\text {Sub }}$. This critical value of $\mathrm{Du}$ increases by the increase of Sr. For the second case, Figure 3(b) indicates that the subcritical convection starts from $D_{C}^{\text {Sub }}$. More increase of $\mathrm{Du}$ from this threshold leads to a linear increase of $R_{T C}^{\text {Sub }}$ and stabilizes more the system. Note that for the case of Figure 3(b), the effect of $\mathrm{Sr}$ on $R_{T C}^{\mathrm{Sub}}$ is seen to be limited, but its effect of the limit $\mathrm{Du}_{C}^{\text {sub }}$ is important. The analytical expression of $\mathrm{Du}_{C}^{\text {sub }}$ is given by $\mathrm{Du}_{C}^{\text {sub }}=\left(2+\beta_{1} \mp \sqrt{\beta_{1}^{2}-4 \beta_{2}}\right) / 2$ SrLe, where $\beta_{1}$ and $\beta_{2}$ are functions of the governing parameters. The opposite tendencies observed by increasing the Dufour parameter in Figures 3(a) and 3(b) are explained by the fact that the increase of Du leads to the successive cross of regions 5 and region 4 in the case of Figure 3(a), while in the case of Figure 3(b) it leads to the successive cross of regions 4 and 5. For $N \leq-$ Le, the subcritical convection corresponding to region 6 appears only for $\mathrm{Sr}<\mathrm{Sr}_{C}$ as shown in Figure 1 . 


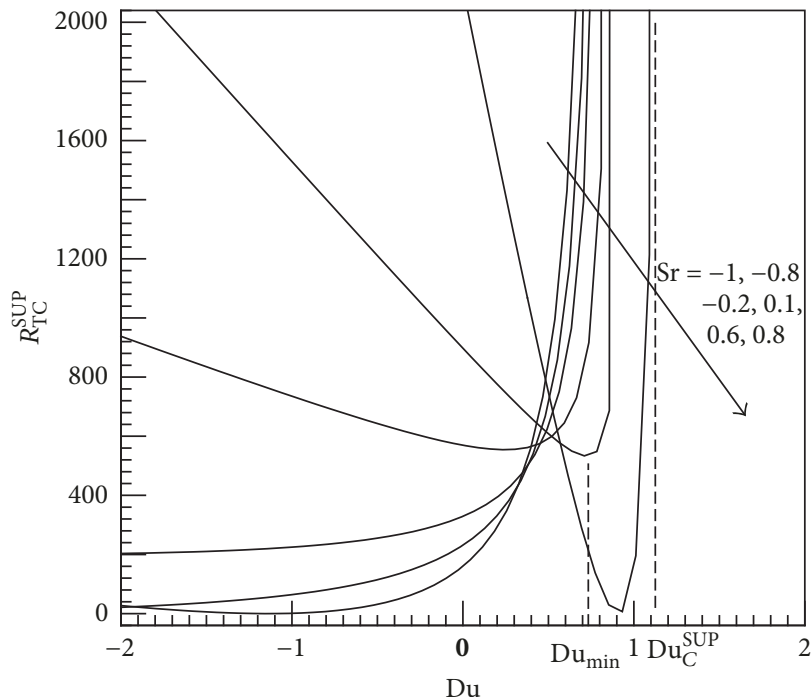

(a) The evolution of $R_{T C}^{\text {sup }}$ with Du for $N=1.5$ and Le $=1.1$ (case of $N \geq N_{L}^{\text {Sup }}$ )

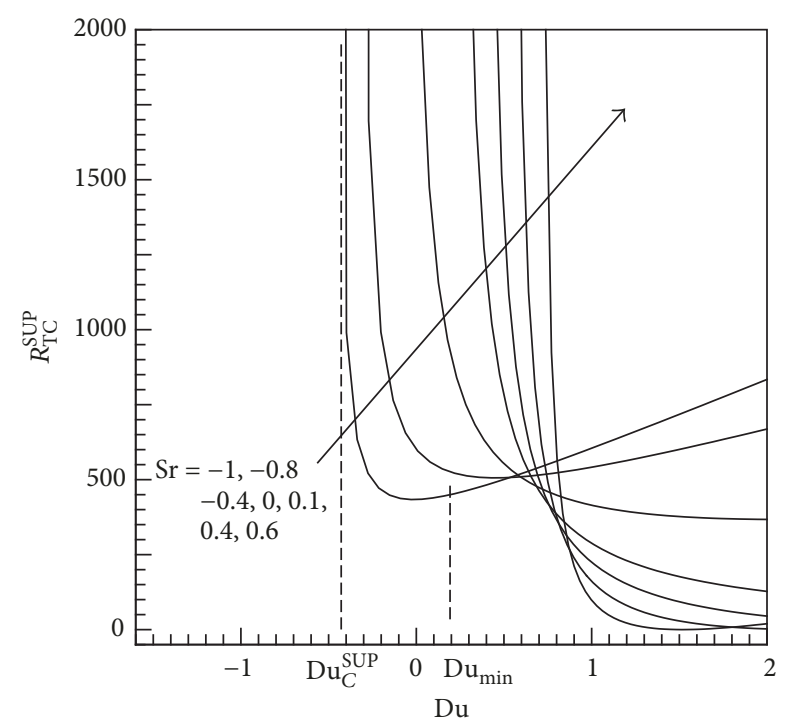

(b) The evolution of $R_{T C}^{\text {sup }}$ with Du for $N=-1.5$ and Le $=1.1$ (case of $N<N_{L}^{\text {Sup }}$ )

Figure 2

For this region, the subcritical convection starts from $D_{u d}$ and increases linearly with Du inducing a stabilizing effect. The Soret parameter has an important effect on the subcritical Rayleigh number in this region as indicated in Figure 3(c).

The buoyancy ratio $N$ measures the relative importance of solutal buoyancy force (due to combined contributions of imposed flux of mass and Soret effect) and thermal buoyancy force (induced by the imposed flux of heat and Dufour effect). It could be varied by changing the imposed flux of heat and/or mass or by considering various fluids with different properties.

The influence of the buoyancy ratio $N$ on the thresholds of stationary convection $R_{\mathrm{TC}}^{\text {Sup }}$ is illustrated in Figures $4(\mathrm{a})$ and 4(b) for Le $=1.1$ and various Sr. In Figure 4(a), Du $=-1.5$ to illustrate the case $\mathrm{Du}<\mathrm{Du}_{L}^{\text {sup }}$, while in Figure 4(b) $\mathrm{Du}=1.5$ to illustrate the case $\mathrm{Du} \geq \mathrm{Du}_{L}^{\text {sup }}$. As shown from Figure 4(a), the evolution of $R_{T C}^{\text {sup }}$ with $N$ changes drastically depending on whether $\mathrm{Du}<\mathrm{Du}_{L}^{\text {sup }}$ or $\mathrm{Du} \geq \mathrm{Du}_{L}^{\text {sup }}$. For $\mathrm{Du} \geq \mathrm{Du}_{L}^{\text {sup }}$, an increase of $N$ from a supercritical value $N_{C}^{\text {sup }}$ induces a destabilizing effect characterized first by a sharp decrease of $R_{T C}^{\text {sup }}$ followed by a monotonous and moderate decrease. The trend is inverted for $\mathrm{Du} \geq \mathrm{Du}_{L}^{\text {sup }}$ as it can be seen in Figure 4(b). In fact, $R_{T C}^{\text {sup }}$ increases first monotonously and in a moderate way. A change in the tendency of the increase occurs when the value of $N$ becomes close to a critical value $N_{C}^{\text {sup }}$, leading to a quick increase toward the infinite value of the asymptote. For this case, it is clear that the increase of $N$ has a stabilizing role. This behavior can be explained by the fact that, for $\mathrm{Du}=-1.5$, case corresponding to $\mathrm{Du}<$ $\mathrm{Du}_{L}^{\text {sup }}$, the increase of $N$ in Figure 1 leads to cross region 1 first which requires an infinite Rayleigh number to trigger the flow. However, for $\mathrm{Du}=1.5$, case corresponding to $\mathrm{Du} \geq \mathrm{Du}_{L}^{\text {sup }}$, region 3 is crossed first, then region 1. The latter corresponds to the case where the parallel flow is impossible, which explains why an infinite Rayleigh number is required to start the flow. The critical value $N_{C}^{\text {sup }}$ can be calculated from the following analytical expression:

$$
N_{C}^{\text {sup }}=-\frac{\operatorname{SrLe}\left(\mathrm{LeDu}-\mathrm{Le}^{2}-\mathrm{Le}\right)+1}{\mathrm{Du}\left(\mathrm{LeSr}-\mathrm{Le}^{2}-\mathrm{Le}\right)+\mathrm{Le}} .
$$

The expression of $\mathrm{Du}_{L}^{\text {Sup }}$ is also calculated analytically to obtain

$$
\operatorname{Du}_{L}^{\text {Sup }}=-\frac{\mathrm{Le}}{1-\operatorname{Le}(\mathrm{Sr}-1)}
$$

The evolution of the subcritical Rayleigh number, $R_{T C}^{\text {Sub }}$, versus $N$ is depicted in Figures 5(a) and 5(b). The case of $\mathrm{Du}<\mathrm{Du}_{L}^{\text {sub }}$ is illustrated in Figure 5(a) for $\mathrm{Du}=-1.5$, $\mathrm{Le}=1.1$, and different values of Sr. It is to note that, for this case, the subcritical flow exists only for negative values of $N$. By increasing $N$ from a subcritical value $N_{C}^{\text {sub }}$, the subcritical Rayleigh number $R_{T C}^{\text {Sub }}$ undergoes a sharp decline from its infinite value, indicating that the increase of $N$ has a destabilizing effect. For this case, by increasing $N$, we cross first region 1 (rest state region) in Figure 1 which explains why an infinite Rayleigh number is required to start the flow for $N=N_{C}^{\text {Sub }}$. The case $\mathrm{Du} \geq \mathrm{Du}_{L}^{\text {Sub }}$ is exemplified in Figure 5(b) for $\mathrm{Du}=2, \mathrm{Le}=1.1$, and different values of Sr. The examination of this figure shows a big change in the behavior compared with the first case illustrated in Figure 5(a). In fact, for $\mathrm{Sr}=-0.8$, an increase of $N$ induces first an increase of $R_{T C}^{\text {Sub }}$ toward a maximum value and undergoes after that a decrease in the remaining range of $N$. Note that, for $\mathrm{Sr}=0.1$, the fluid remains at rest as long as $N<N_{C}^{\text {Sub }}$. 


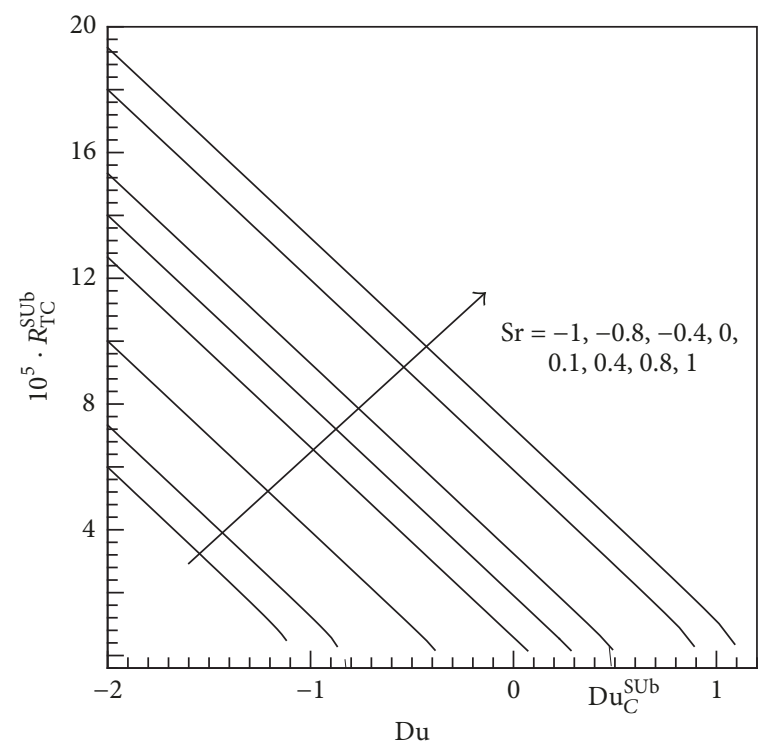

(a) The evolution of $R_{T C}^{\text {Sub }}$ versus Du for $N=-1$ and $\mathrm{Le}=1.1$ (case of - Le $<N \leq N_{L}^{\text {Sub }}$ )

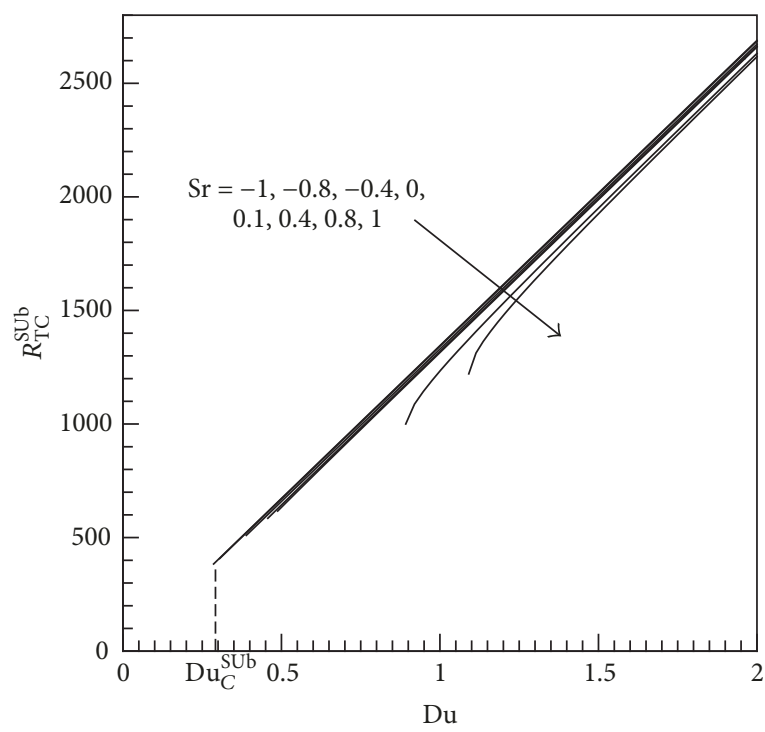

(b) The evolution of $R_{T C}^{\text {Sub }}$ versus Du for $N=1.5$ and Le $=1.1$ (case of $N>N_{L}^{\text {Sub }}$ )

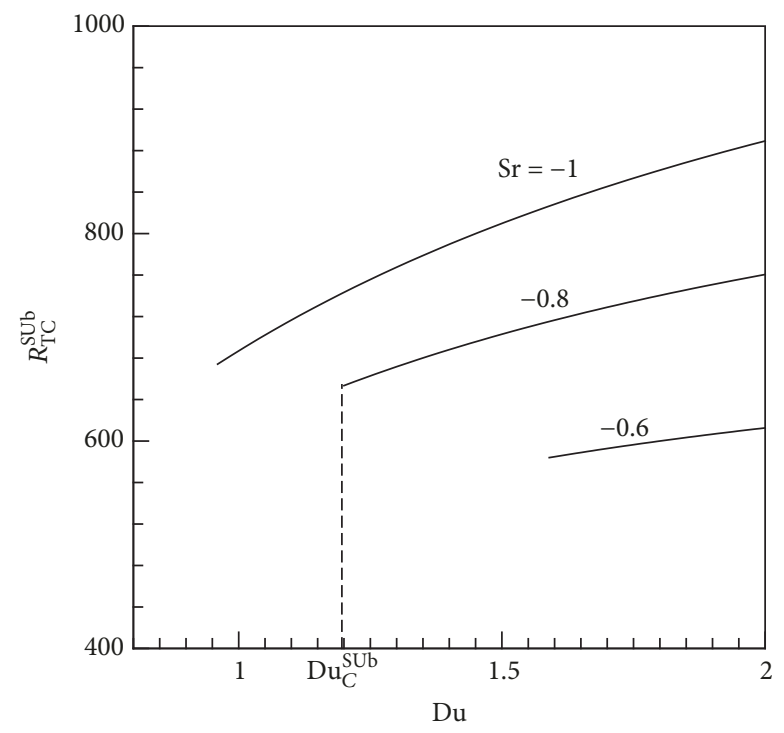

(c) The evolution of $R_{T C}^{\text {Sub }}$ with Du for $N=-1.2$ and Le $=1.1$ (case of $N \leq-\mathrm{Le}$ )

Figure 3

The analytical expressions of $N_{C}^{\text {Sub }}$ and $\mathrm{Du}_{L}^{\text {Sub }}$ are, respectively, given by

$$
\begin{aligned}
N_{C}^{\mathrm{SuB}} & =\frac{B \operatorname{SrLe}\left(\mathrm{LeDu}-\mathrm{Le}^{2}-\mathrm{Le}\right)+B-A^{2} \mathrm{Le}^{2}}{A^{2} \mathrm{Le}-B \mathrm{Du}(\mathrm{SrLe}-\mathrm{Le}-1)-B \mathrm{Le}}, \\
\mathrm{Du}_{\mathrm{C}}^{\mathrm{sub}} & =\frac{2+\alpha_{1} \mp \sqrt{\alpha_{1}^{2}-4 \alpha_{2}}}{2 \mathrm{SrLe}},
\end{aligned}
$$

where $\alpha_{1}=\left((\mathrm{LeSr}-\mathrm{Le}-1)\left(\mathrm{Le}^{2}+4 \mathrm{Le}+1\right)+2 \mathrm{SrLe}^{3}\right) / \mathrm{Le}(2 \mathrm{Le}+$ $2-\mathrm{SrLe})$ and $\alpha_{2}=-(\mathrm{Le}+1)^{3}(\mathrm{SrLe}-1) / \mathrm{Le}(2 \mathrm{Le}+2-\mathrm{SrLe})$.
4.2. Effect of Du on the Flow Intensity and Heat and Mass Transfer. In this subsection, the influence of the Dufour effect on the flow intensity and heat and mass transfer is examined. The effect of $\mathrm{Du}$ on $\psi_{0}, \mathrm{Nu}$, and $\mathrm{Sh}$ is depicted in Figures 6(a)-6(c) for Le $=1.1, A_{r}=10$, different values of $\mathrm{Sr}$, and two cases: $R_{T}=2730$ and $N=1.2$ (case I) and $R_{T}=$ 889.3, $N=-1.2$ (case II). The choice of these two cases was dictated by the fact that, by varying $\mathrm{Du}$, two types of traversed regions are possible depending on whether $N \geq$ - Le (case I) or $N<-$ Le (case II). In these figures, it can be seen that the numerical results are in excellent agreement with the analytical ones corresponding to the stable branches. 


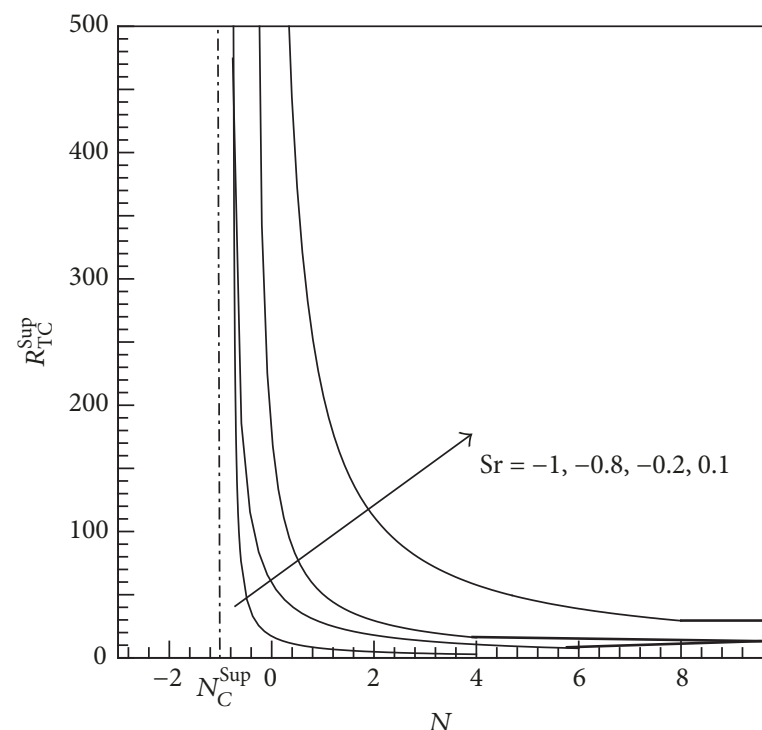

(a) The evolution of $R_{T C}^{\text {sup }}$ with $N$ for $\mathrm{Du}=-1.5$ and $\mathrm{Le}=1.1$ (case of $\mathrm{Du}<\mathrm{Du}_{L}^{\text {sup }}$ )

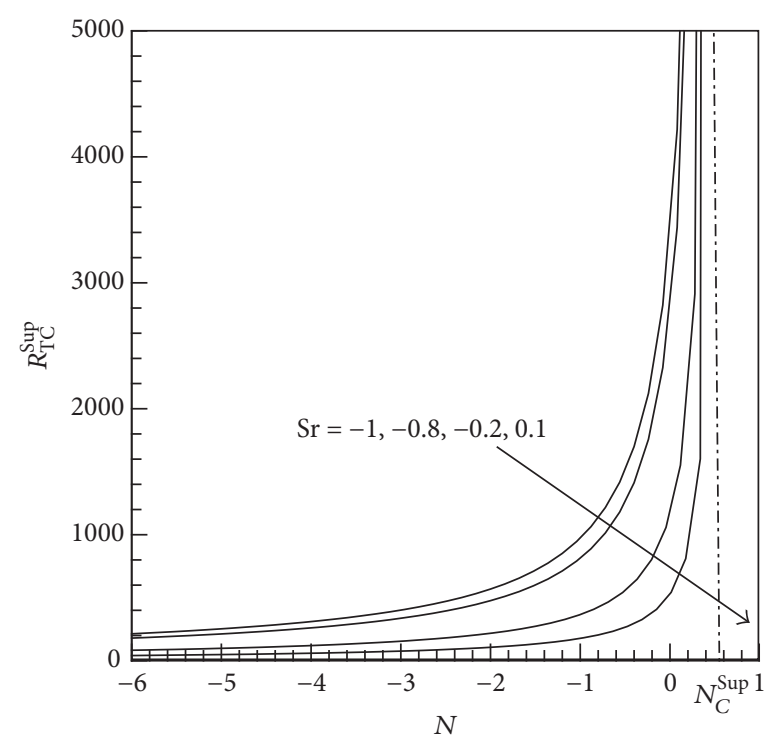

(b) The evolution of $R_{T C}^{\text {sup }}$ with $N$ for $\mathrm{Du}=1.5$ and Le $=1.1$ (case of $\mathrm{Du} \geq \mathrm{Du}_{L}^{\text {sup }}$ )

Figure 4

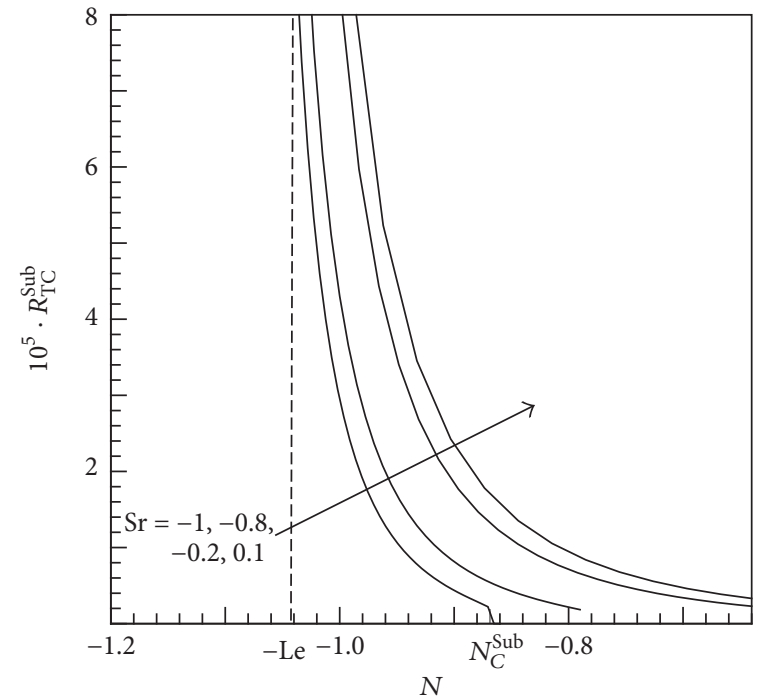

(a) The evolution of $R_{T C}^{\text {sub }}$ with $N$ for $\mathrm{Du}=-1.5$ and $\mathrm{Le}=1.1$ (case of $\mathrm{Du}<\mathrm{Du}_{L}^{\text {sub }}$ )

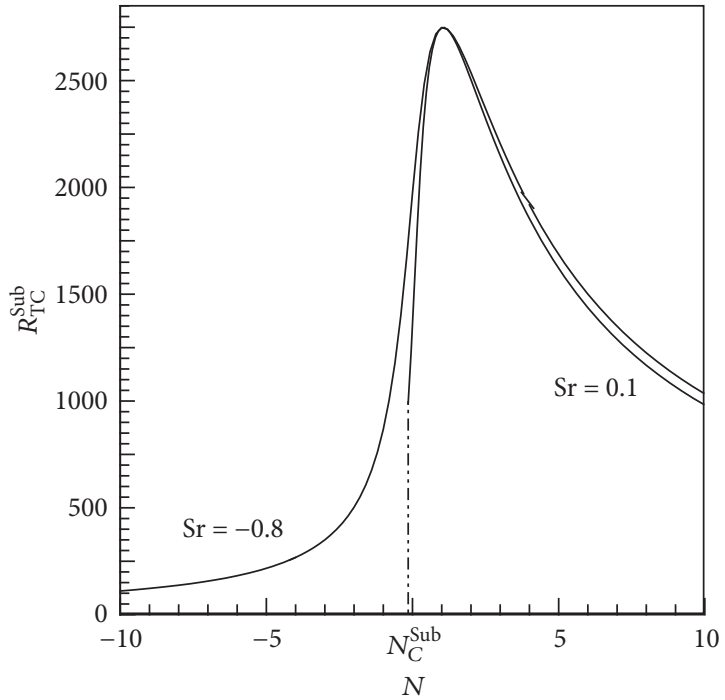

(b) The evolution of $R_{T C}^{\text {sub }}$ versus $N$ for $\mathrm{Du}=2$ and Le $=1.1$ (case of $\mathrm{Du} \geq \mathrm{Du}_{L}^{\mathrm{sub}}$ )

Figure 5

For case I, both stable and unstable branches are existing regardless of the Soret parameter. For the stable branch, the evolutions of $\psi_{0}$ and $\mathrm{Nu}$ versus $\mathrm{Du}$ are characterized by a monotonous decrease (the decrease occurs with a higher rate for the negative value of $\mathrm{Sr}$ ) and vanishes when Du exceeds a critical value $\mathrm{Du}_{c}$ which depends on $\mathrm{Sr}$. The unstable branch exists only between two critical values of $\mathrm{Du}$, that is, for $\mathrm{Du}_{u}<\mathrm{Du} \leq \mathrm{Du}_{c}$. Within this range of $\mathrm{Du}$, the evolution of $\psi_{0}$ is characterized by an important increase toward a maximum reached for $\mathrm{Du}=\mathrm{Du}_{c}$. This critical value $\mathrm{Du}_{c}$ marks also the limit of the existence of the unstable branch.
This means that, before their disappearance, the unstable solutions become identical to the ones corresponding to the stable branches.

For case II, the evolutions of $\psi_{0}$ and $\mathrm{Nu}$ versus $\mathrm{Du}$ depend on the Soret parameter. In fact, for $\mathrm{Sr}=-1$ (for this value of $\mathrm{Sr}$ the corresponding regions in $\mathrm{N}$-Du plane are not presented here), both stable and unstable solutions exist and the quantities $\psi_{0}$ and $\mathrm{Nu}$ increase with $\mathrm{Du}$ to reach a maximum and decrease afterward before vanishing. The unstable branch exists for $\mathrm{Du}_{u}<\mathrm{Du} \leq \mathrm{Du}_{c}$. For $\mathrm{Sr}=0.1$, only the stable solution exists and the quantities $\psi_{0}$ and $\mathrm{Nu}$ 


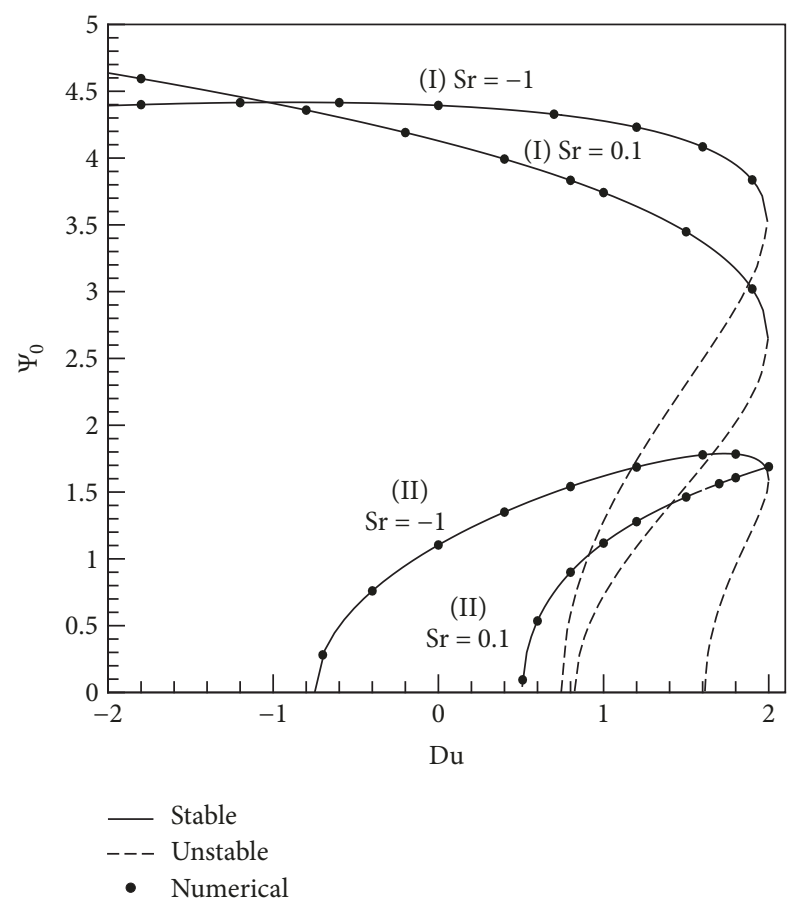

(a) Influence of the Dufour number on $\psi_{0}$ for $\mathrm{Le}=1.1$ and $\mathrm{Ar}=10$ : (I) $R_{T}=2730$ and $N=1.2$ (case of $N \geq-$ Le); (II) $R_{T}=889$ and $N=-1.2($ case of $N<-$ Le $)$

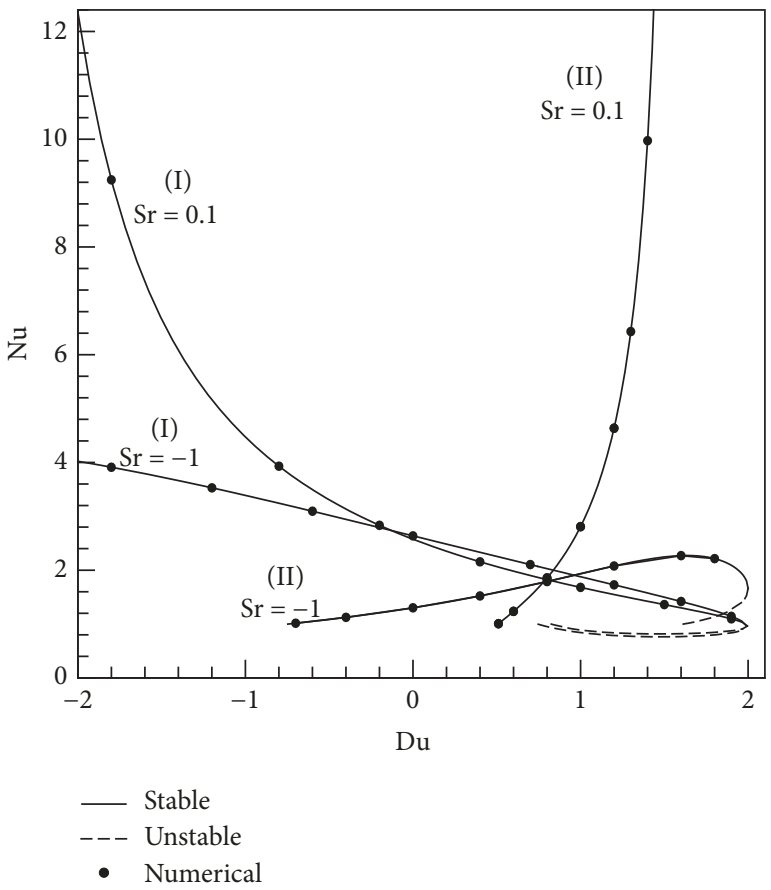

(b) Influence of the Dufour number on $\mathrm{Nu}$ for $\mathrm{Le}=1.1$ and $\mathrm{Ar}=10$ : (I) $R_{T}=2730$ and $N=1.2$ (case of $N \geq-\mathrm{Le}$ ); (II) $R_{T}=889$ and $N=-1.2($ case of $N<-$ Le $)$

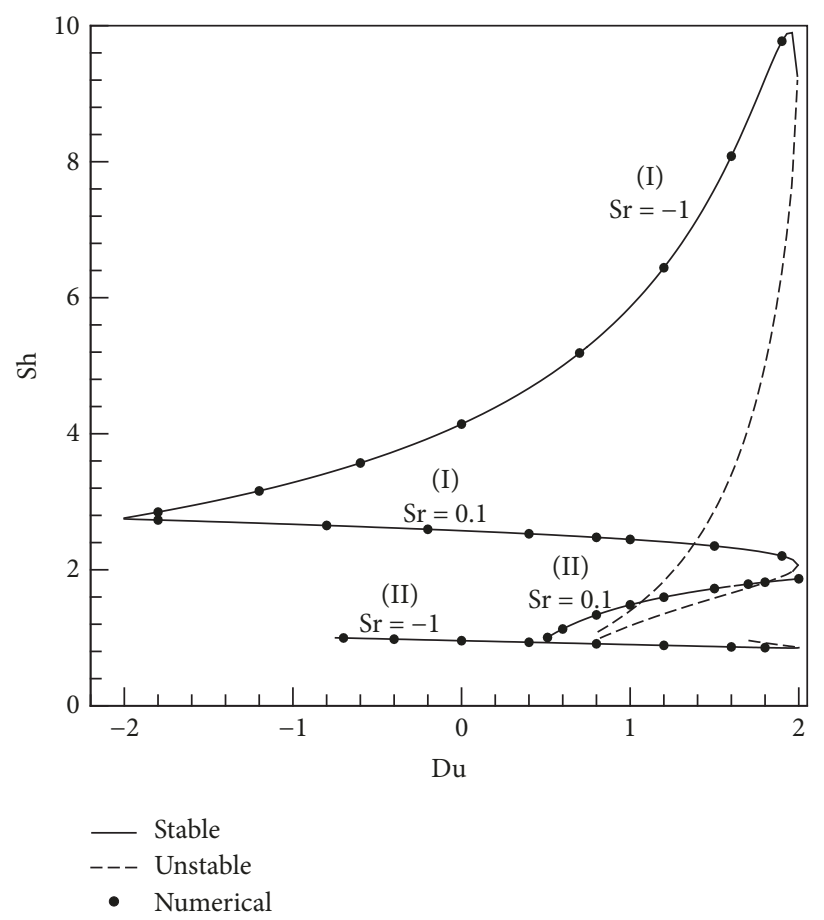

(c) Influence of the Dufour number on Sh for Le $=1.1$ and $\mathrm{Ar}=10$ : (I) $R_{T}=2730$ (case of $N \geq-$ Le); (II) $R_{T}=890($ case of $N<-$ Le)

Figure 6

exhibit monotonous increases with Du. The dependence of the flow intensity vis-à-vis $\mathrm{Sr}$ can be explained by the fact that, by varying $\mathrm{Du}$, the crossed regions are region 2 and then region 6 for $\mathrm{Sr}=-1$ while for $\mathrm{Sr}=0.1$ the crossed regions are region 1 and then region 2. From these observations, we can conclude that the Dufour effect enhances/reduces the flow intensity and heat transfer for $N<-$ Le $/ N \geq-$ Le.

The evolution of Sh versus Du exhibits a different tendency in comparison with $\psi_{0}$ and $\mathrm{Nu}$. In fact, the Sh variations with Du show similar tendencies independently of 
the crossed regions for both cases I and II and $\mathrm{Sr}=-1$. For this case, Sh varies slightly with $\mathrm{Du}$. The behavior changes for $\mathrm{Sr}=0.1$ since the evolution of $\mathrm{Sh}$ is characterized by a monotonous increase with $\mathrm{Du}$ to reach a maximum marking the limit of the stable branch. For case I, by increasing Du the crossed regions are successively regions 3,4 , and 5 while for case II the crossed regions are 2 (crossed first) and 6.

\section{Conclusion}

Thermosolutal natural convection within a shallow fluid layer subjected to vertical gradients of temperature and solute is investigated analytically and numerically in the presence of Soret and Dufour effects. The analytical solution based on the parallel flow assumption is found to be in good agreement with the numerical solution based on a finite-difference method used to solve the full governing equations in their transient forms. In view of the results discussed, the main conclusions are as follows:

(i) The thresholds of stationary and subcritical convections are derived analytically versus the governing parameters.

(ii) We have demonstrated analytically that the plane $N$ Du can be divided into different regions (up to six regions) with specific flow regimes.

(iii) The number of these regions, their extension, and their locations depend on whether the product $\mathrm{SrDu}=f(\mathrm{Le})$ is lower or higher than $-\left(1+\mathrm{Le}^{2}\right) / 2 \mathrm{Le}^{2}$.

(iv) The influence of the Dufour effect on the thresholds of stationary and subcritical convections may be stabilizing or destabilizing, depending on the buoyancy ratio and the Soret parameter.

(v) The important effect of the thermodiffusion and diffusion-thermo on fluid flow and heat and mass transfer characteristics is well demonstrated.

\section{Nomenclature}

$A_{r}$ : Aspect ratio of the enclosure $\left(=L^{\prime} / H^{\prime}\right)$

Du: Dufour parameter $\left(=\beta_{T} \kappa_{12} / \beta_{S} \kappa_{11}\right)$

$g$ : Gravitational acceleration

$H^{\prime}$ : Height of the enclosure

$J^{\prime}$ : Constant mass flux per unit area

$L^{\prime}$ : Length of the enclosure

Le: Lewis number $\left(=\kappa_{11} / \kappa_{22}\right)$

$N$ : Buoyancy ratio $\left(=\beta_{S} \Delta S^{\prime} / \beta_{T} \Delta T^{\prime}\right)$

$\mathrm{Nu}$ : Thermal Nusselt number

$q^{\prime}$ : Constant heat flux per unit area

Pr: Prandtl number $\left(=\nu / \kappa_{11}\right)$

$R_{T}$ : Thermal Rayleigh number $\left(=g \beta_{T} \Delta T^{\prime} H^{\prime 3} / \kappa_{11} \nu\right)$

$S: \quad$ Dimensionless solute concentration $\left(=\left(S^{\prime}-S_{0}^{\prime}\right) / \Delta S^{\prime}\right)$

$\Delta S^{\prime}$ : Concentration solute difference $\left(=j^{\prime} H^{\prime} / \kappa_{22} \rho C_{p}\right)$

Sr: Soret parameter $\left(=\beta_{S} \kappa_{21} / \beta_{T} \kappa_{11}\right)$

Sh: Sherwood number
T: Dimensionless temperature $\left(=\quad\left(T^{\prime}-\right.\right.$ $\left.\left.T_{0}^{\prime}\right) / \Delta T^{\prime}\right)$

$\Delta T^{\prime}$ : Temperature difference $\left(=q^{\prime} H^{\prime} / \kappa_{11} \rho C_{p}\right)$

$t$ : Dimensionless time $\left(=t^{\prime} / H^{\prime 2} / \kappa_{11}\right)$

$u$ : Dimensionless horizontal velocity $\left(=u^{\prime} H^{\prime} / \kappa_{11}\right)$

$v$ : Dimensionless vertical velocity $\left(=v^{\prime} H^{\prime} / \kappa_{11}\right)$

$x$ : Dimensionless distance along the $x$-axis (= $\left.x^{\prime} / H^{\prime}\right)$

$y$ : Dimensionless distance along the $y$-axis (= $\left.y^{\prime} / H^{\prime}\right)$

Greek Symbols

$\beta_{S}: \quad$ Solute expansion coefficient

$\beta_{T}$ : Thermal expansion coefficient

$\mathrm{K}_{11}$ : Thermal diffusivity

$\mathrm{K}_{12}$ : Cross-diffusion due to $S$-component

$\mathrm{K}_{21}$ : Cross-diffusion due to $T$-component

$\kappa_{22}$ : Solute diffusivity

$\rho C p$ : Heat capacity

$v$ : Kinematic viscosity of the fluid

$\Psi: \quad$ Dimensionless stream function, $\Psi^{\prime} / \kappa_{11}$

Subscripts

c: Critical value

0: Reference value.

Superscripts

I: Dimensional variables

Sub: Subcritical

Sup: Supercritical.

\section{Conflicts of Interest}

The authors declare that there are no conflicts of interest regarding the publication of this paper.

\section{References}

[1] O. E. Tewfikf and Y. Ji-Wu, "The thermodynamic coupling between heat and mass transfer in free convection with helium injection," International Journal of Heat and Mass Transfer, vol. 6, no. 10, pp. 915-923, 1963.

[2] D. A. Nield and A. Bejan, Convection in Porous Media, vol. 3rd, Spring, New York, NY, USA, 2006.

[3] S. R. De Groot and P. Mazur, Non-Equilibrium Thermodynamics, Dover Publications, New York, NY, USA, 1984.

[4] M. Ouriemi, P. Vasseur, A. Bahloul, and L. Robillard, "Natural convection in a horizontal layer of a binary mixture," International Journal of Thermal Sciences, vol. 45, no. 8, pp. 752-759, 2006.

[5] M. Er-Raki, M. Hasnaoui, A. Amahmid, and M. Bourich, "Subcritical convection in the presence of Soret effect within a horizontal porous enclosure heated and salted from the short sides," International Journal of Numerical Methods for Heat \& Fluid Flow, vol. 21, no. 2, pp. 150-167, 2011. 
[6] A. Amahmid, M. Hasnaoui, M. Mamou, and P. Vasseur, "Double-diffusive parallel flow induced in a horizontal Brinkman porous layer subjected to constant heat and mass fluxes: Analytical and numerical studies," Wärme- und Stoffübertragung, vol. 35, no. 5, pp. 409-421, 1999.

[7] M. A. Rahman and M. Z. Saghir, "Thermodiffusion or Soret effect: historical review," International Journal of Heat and Mass Transfer, vol. 73, pp. 693-705, 2014.

[8] M. Bourich, M. Hasnaoui, M. Mamou, and A. Amahmid, "Soret effect inducing subcritical and Hopf bifurcations in a shallow enclosure filled with a clear binary fluid or a saturated porous medium: a comparative study," Physics of Fluids, vol. 16, no. 3, pp. 551-568, 2004.

[9] M. S. Malashetty, "Anisotropic thermoconvective effects on the onset of double diffusive convection in a porous medium," International Journal of Heat and Mass Transfer, vol. 36, no. 9, pp. 2397-2401, 1993.

[10] R. G. Mortimer and H. Eyring, "Elementary transition state theory of the Soret and Dufour effects," Proceedings of the National Acadamy of Sciences of the United States of America, vol. 77, no. 4, pp. 1728-1731, 1980.

[11] S. N. Gaikwad, M. S. Malashetty, and K. Rama Prasad, "Linear and non-linear double diffusive convection in a fluid-saturated anisotropic porous layer with cross-diffusion effects," Transport in Porous Media, vol. 80, no. 3, pp. 537-560, 2009.

[12] N. Nithyadevi and R. J. Yang, "Double diffusive natural convection in a partially heated enclosure with Soret and Dufour effects," International Journal of Heat and Fluid Flow, vol. 30, no. 5, pp. 902-910, 2009.

[13] O. D. Makinde, K. Zimba, and O. A. Bég, "Numerical study of chemically-reacting hydromagnetic boundary layer flow with soret/dufour effects and a convective surface boundary condition," International Journal of Thermal and Environmental Engineering, vol. 4, no. 1, pp. 89-98, 2012.

[14] D. Pal and H. Mondal, "Effects of Soret Dufour, chemical reaction and thermal radiation on MHD non-Darcy unsteady mixed convective heat and mass transfer over a stretching sheet," Communications in Nonlinear Science and Numerical Simulation, vol. 16, no. 4, pp. 1942-1958, 2011.

[15] C.-Y. Cheng, "Soret and Dufour effects on natural convection boundary layer flow over a vertical cone in a porous medium with constant wall heat and mass fluxes," International Communications in Heat and Mass Transfer, vol. 38, no. 1, pp. 44-48, 2011.

[16] R. Tsai and J. S. Huang, "Heat and mass transfer for Soret and Dufour's effects on Hiemenz flow through porous medium onto a stretching surface," International Journal of Heat and Mass Transfer, vol. 52, no. 9-10, pp. 2399-2406, 2009.

[17] T. Hayat, M. Mustafa, and I. Pop, "Heat and mass transfer for Soret and Dufour's effect on mixed convection boundary layer flow over a stretching vertical surface in a porous medium filled with a viscoelastic fluid," Communications in Nonlinear Science and Numerical Simulation, vol. 15, no. 5, pp. 1183-1196, 2010.

[18] J. Wang, M. Yang, and Y. Zhang, "Onset of double-diffusive convection in horizontal cavity with Soret and Dufour effects," International Journal of Heat and Mass Transfer, vol. 78, pp. 1023-1031, 2014.

[19] A. Lagra, M. Hasnaoui, A. Amahmid, and M. Bourich, "Double diffusive convection in a shallow horizontal binary fluid in the presence of Soret and Dufour effects," in Proceedings of the 12ème Congrès de Mécanique, Casablanca, Morocco, April 2015. 


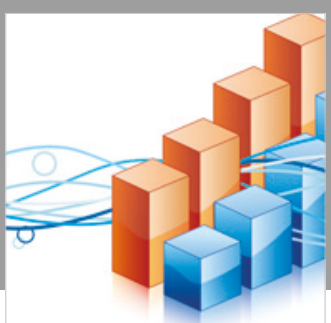

Advances in

Operations Research

\section{-n-m}
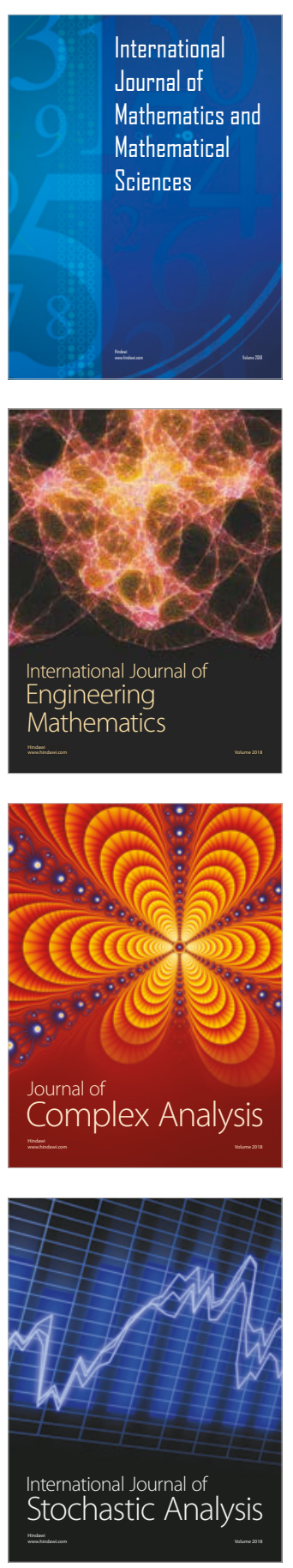
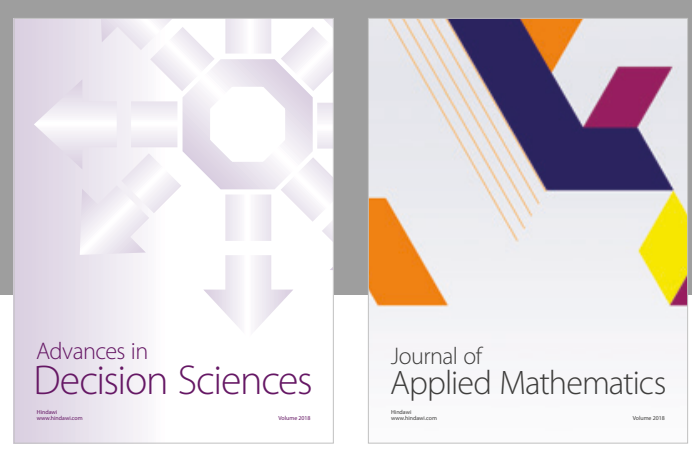

Journal of

Applied Mathematics
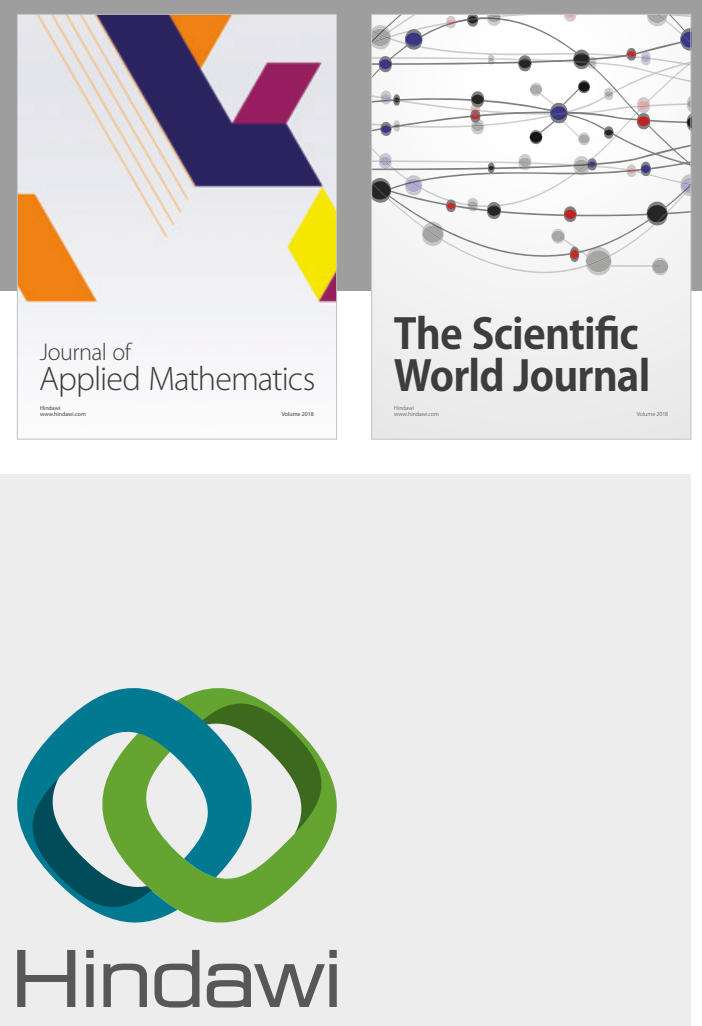

Submit your manuscripts at

www.hindawi.com

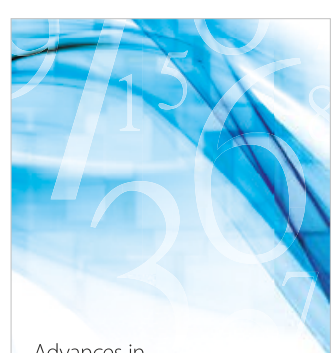

Advances in
Numerical Analysis
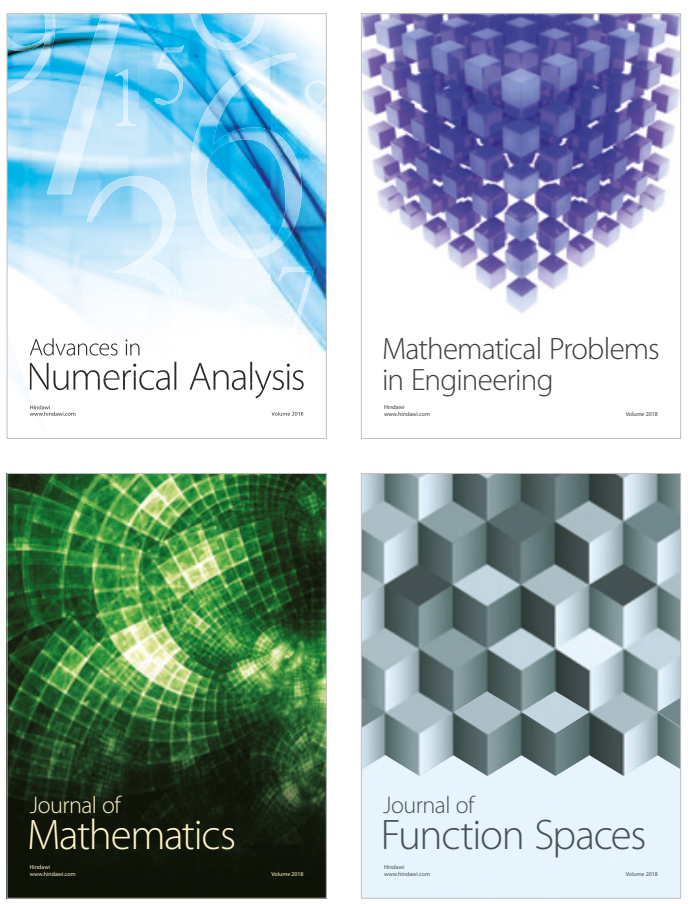

Mathematical Problems in Engineering

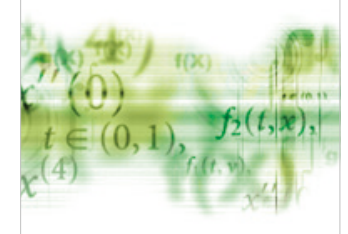

International Journal of

Differential Equations

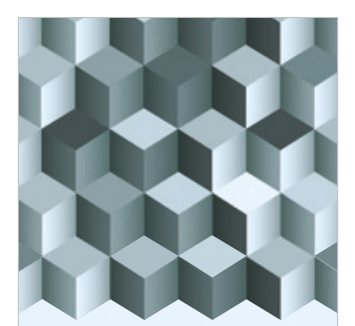

Journal of

Function Spaces

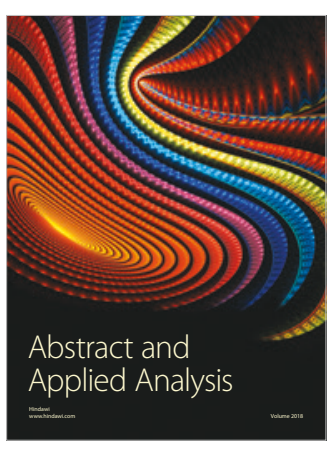

The Scientific

World Journal

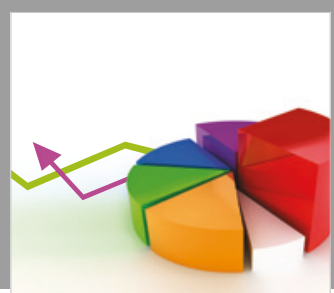

Journal of

Probability and Statistics
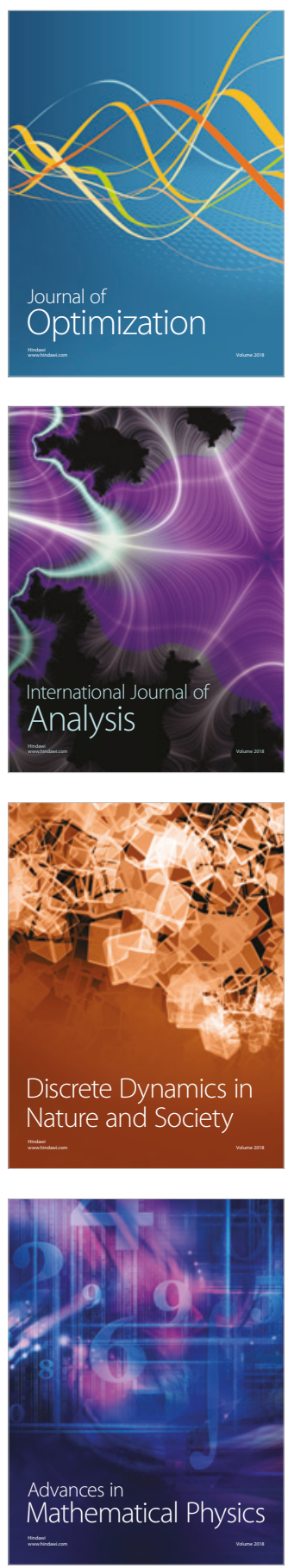